\title{
Spontaneous subcapsular renal haematoma
}

\section{D) Solomon} MBChB, FFRad(D)SA

Constantiaberg Medi-Clinic, Plumstead, Cape Town

\author{
Abstract \\ Spontaneous \\ subcapsular renal \\ haematoma is a rare \\ condition caused by \\ bleeding into the \\ subcapsular or \\ perinephric space in \\ the absence of \\ trauma, bleeding \\ diathesis or anti- \\ coagulation. If the \\ contralateral kidney is \\ normal, radical \\ nephrectomy is
}

recommended

due to a high

incidence of

small occult

tumours.

\section{Case report}

A 74-year-old man presented with a three day history of left loin pain radiating to the groin. The pain was not typically colicky in nature and there were no associated constitutional symptoms. He had been previously well and besides mild hypertension, there was no past medical history of note. There was no history of trauma and he was not taking anti-coagulant therapy. Clinically, he was noted to be undistressed, apyrexial and normotensive. The left kidney was felt to be slightly enlarged and $1+$ haematuria was noted. His general practitioner suspected renal colic and he was referred for an excretory urogram. There were no apparent calcifications on the survey and the right kidney, collecting system and ureter appeared normal. On the left there was no identifiable nephrogram and no excretion was noted at 1 hour. An ultrasound examination revealed a $10 \mathrm{~cm}$ solid appearing left renal mass replacing almost the entire left kidney, with only a small rim of renal tissue noted anteriorly. This was thought to represent a large renal tumour and computed tomography (CT) was performed approximately four hours after the excretory urogram. On the precontrast images, a persistent nephrogram was noted in the compressed and anteriorly displaced kidney. This was due to a $10 \mathrm{~cm}$ subcapsular mass showing minor extension into the pararenal spaces (Figures 1-2). No focal abnormality was seen in relation

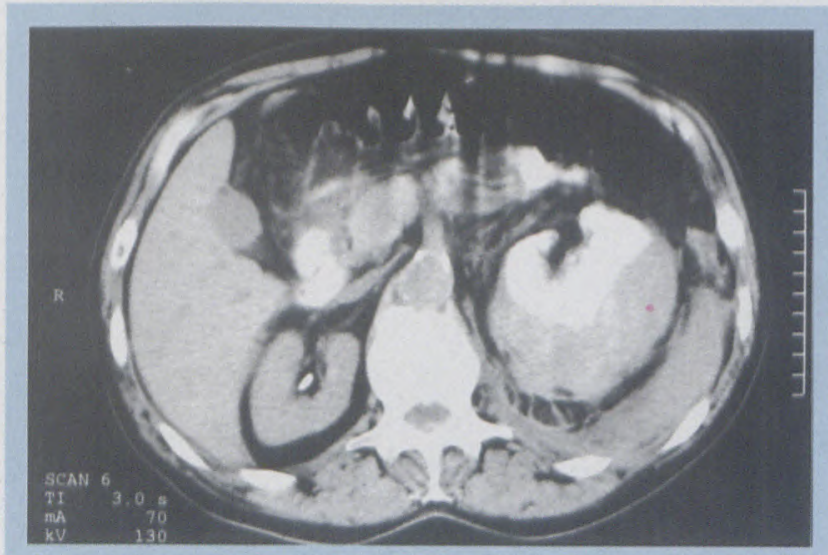

Figure 1: Image obtained four hours after IVU showed persistent left nephrogram with kidney compressed and anteriorly displaced by large subcapsular haematoma. Note extension into posterior pararenal space. to the renal parenchyma and the right kidney appeared normal. A large perinephric haematoma was suspected 


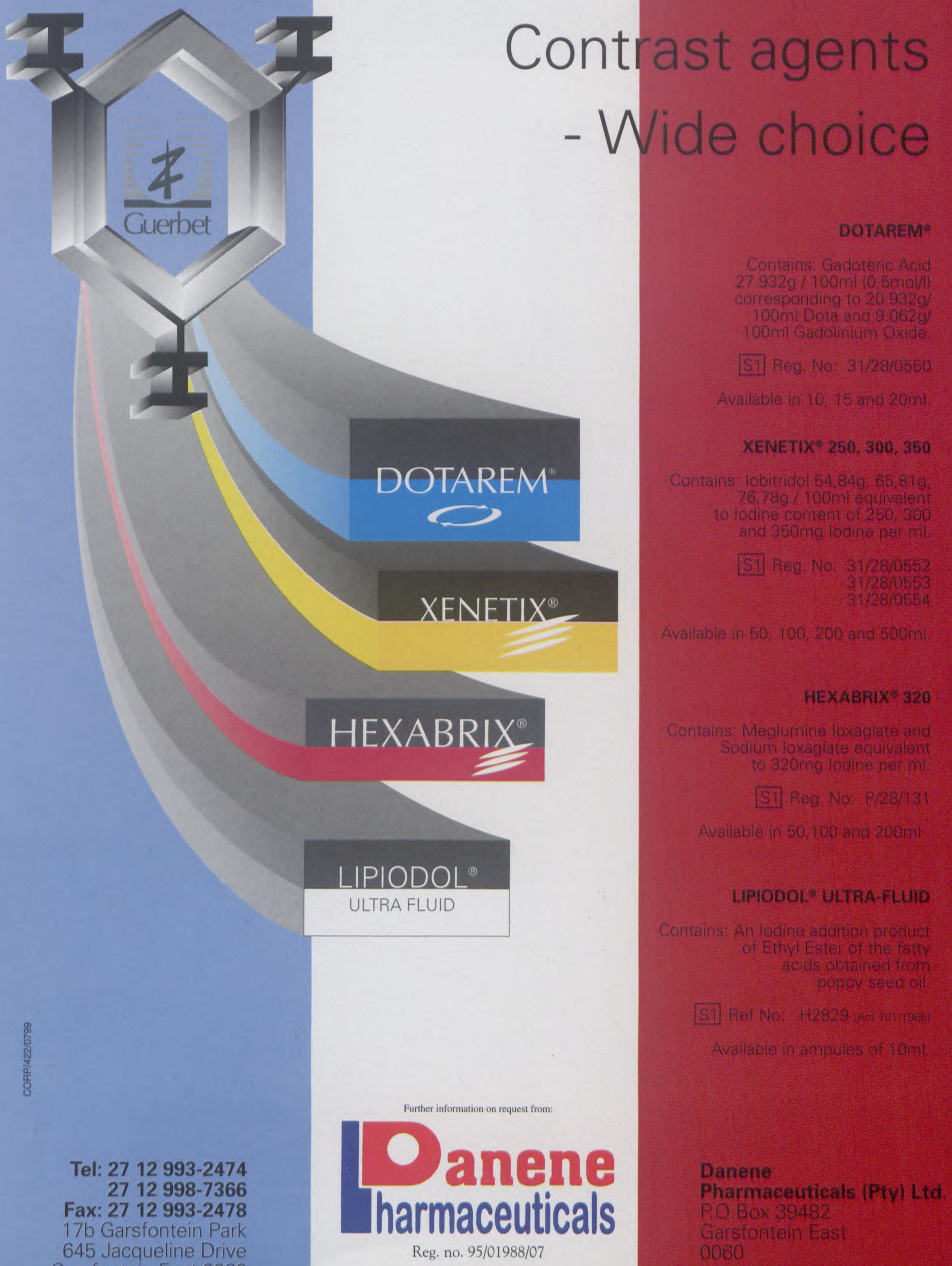


from page 18

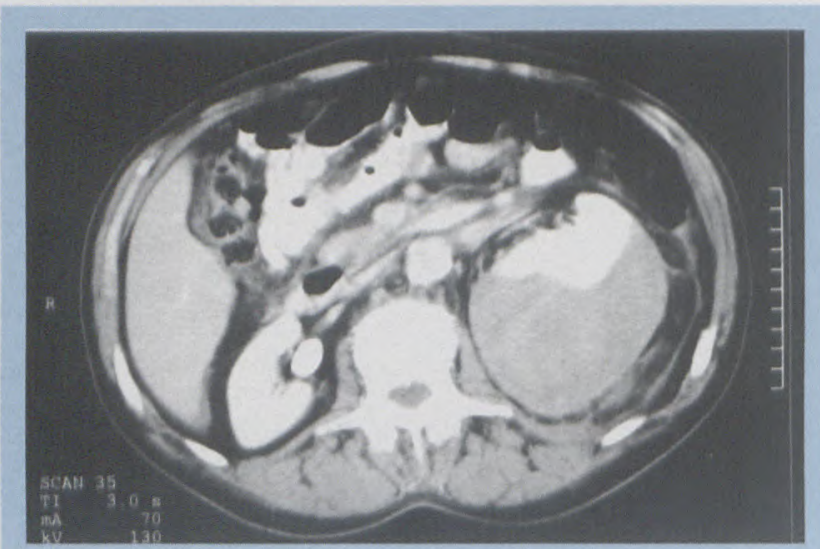

Figure 2: Post contrast showing compression of renal parenchyma. nephrotomography may reveal a displaced kidney with the parenchyma compressed by haematoma. Ultrasound findings are usually non-specific and differentiation of a renal mass from a nephric haematoma may be difficult. CT scan is the investigation of choice and will reveal

and confirmed surgically. A radical nephrectomy was performed and close histological examination of the resected kidney failed to reveal a cause for the bleeding. Post-operative course was uneventful and the patient remains well.

\section{Discussion}

Spontaneous subcapsular or perinephric haematoma is an uncommon clinical problem and occurs in the absence of anti-coagulation, arteritis or trauma. The clinical presentation is variable and depends on the severity and duration of bleeding. This may be sudden with acute onset of upper abdominal or flank pain, nausea and vomiting or subacute, as in our patient, with symptoms suggestive of renal colic. A low-grade temperature may be present and urinalysis is usually normal. Excretory urography with an abnormal soft tissue mass confined by the renal capsule and compressing the renal parenchyma. This may extend into the perinephric and pararenal spaces with effacement of the normal retroperitoneal structures.

Renal angiography to exclude vascular lesions such as arteritis, arteriosclerotic aneurysms, arteriovenous malformations or a small renal cell carcinoma may reveal flattening of the renal parenchyma and displacement of the capsular vessels.

Of the eight cases reported by Kendall et al' six were shown after nephrectomy to have small peripheral carcinomas. Only one of these had suspicious angiographic findings whereas the other five tumours were undetectable pre-operatively. In the other two cases no cause was found. Pollack and Popky ${ }^{2}$ reported small peripheral tumours in five of nine patients with spontaneous subcapsular haematoma, four of which measured less than $1 \mathrm{~cm}$ in diameter.

Due to this high incidence of small peripheral carcinomas, radical nephrectomy with meticulous histological examination of the resected specimen is the recommended treatment. However, pre-operative angiography is considered warranted to exclude vascular disease which may affect the contralateral kidney and prompt a more conservative approach.

\section{Conclusion}

The diagnosis of spontaneous subcapsular haematoma of the kidney is best made with $\mathrm{CT}$, showing a subcapsular mass compressing the renal parenchyma and often extending into the pararenal space. Treatment is usually radical nephrectomy due to the high incidence of small peripheral carcinomas. Pre-operative angiography, although unreliable in the exclusion of small tumours is indicated to exclude other vascular causes which may prompt a more conservative approach.

\section{References}

1. Kendall RA, Senay BA, Coll ME Spontaneous subcapsular renal haematoma: diagnosis and management. Journal of Urology 1988, 139: 246-250.

2. Pollack HM, Popky GL. Roentgenographic manifestations of spontaneous renal haemorrhage: Radiology 1974, 110:1. 